\title{
Multilevel Fast-Multipole Algorithm for Scattering from Conducting Targets Above or Embedded in a Lossy Half Space
}

\author{
Norbert Geng, Member, IEEE, Anders Sullivan, Member, IEEE, and Lawrence Carin, Senior Member, IEEE
}

\begin{abstract}
An extension of the multilevel fast multipole algorithm (MLFMA), originally developed for targets in free space, is presented for the electromagnetic scattering from arbitrarily shaped three-dimensional (3-D), electrically large, perfectly conducting targets above or embedded within a lossy half space. We have developed and implemented electric-field, magnetic-field, and combined-field integral equations for this purpose. The nearby terms in the MLFMA framework are evaluated by using the rigorous half-space dyadic Green's function, computed via the method of complex images. Non-nearby (far) MLFMA interactions, handled efficiently within the multilevel clustering construct, employ an approximate dyadic Green's function. This is expressed in terms of a direct-radiation term plus a single real image (representing the asymptotic far-field Green's function), with the image amplitude characterized by the polarization-dependent Fresnel reflection coefficient. Examples are presented to validate the code through comparison with a rigorous method-of-moments (MoM) solution. Finally, results are presented for scattering from a model unexploded ordnance (UXO) embedded in soil and for a realistic 3-D vehicle over soil.
\end{abstract}

Index Terms-Fast algorithms, method of moments (MoM), numerical methods, scattering.

\section{INTRODUCTION}

$\mathbf{E}$ LECTROMAGNETIC scattering from surface and subsurface targets has constituted a problem of long-term interest. Considering numerical modeling of such problems, there has been interest in integral-equation [1]-[3] and differential-equation [4]-[6] based methods. Investigators have considered method-of-moments (MoM) [1]-[3], [7]-[9] and finite-element method (FEM) [6], [9], [10] solutions. The principal focus in the context of time-domain differential-equation solvers has been the finite difference time domain method (FDTD) [4], [5], [11] and more recently, the multiresolution time domain method (MRTD) [12], [13]. Each of these numerical techniques has its relative strengths and weaknesses, and each is applicable to relatively small targets (with characteristic target dimensions small relative to a few wavelengths). There are many applications for which the target may comprise a large number of wavelengths, for example, scattering from vehicles, trees, and large unexploded ordnance. The aforementioned MoM, FEM, FDTD and MRTD algorithms can handle such

Manuscript received August 31, 1999; revised March 27, 2000.

N. Geng is with the Institut für Höchstfrequenztechnik und Elektronik, University of Karlsruhe, Karlsruhe, Germany.

A. Sullivan and L. Carin are with the Department of Electrical and Computer Engineering, Duke University, Durham, NC 27708-0291 USA

Publisher Item Identifier S 0196-2892(00)05895-2. targets in principle, but memory requirements and computation time become excessive.

There has consequently been significant interest recently in the development of a new generation of fast algorithms to cover the range of frequencies for which the above computational tools are inappropriate and for which high-frequency asymptotic techniques (e.g., PO, GTD, and UTD) are not applicable or are difficult to implement. For example, the fast multipole method (FMM) was extended to the case of electromagnetic fields by Rhoklin and his colleagues [14]. More recently, Chew and colleagues have implemented the FMM [15] and extended it to a multilevel framework [16], resulting in the multilevel fast multipole algorithm (MLFMA) [17]-[20]. The MLFMA has been developed for electric-field, magnetic-field, and combined-field integral equations for both perfectly conducting [17]-[19] and dielectric targets [20].

The FMM and MLFMA exploit a particular expansion of the free-space Green's function [14], [15], [21] and therefore, virtually all implementations to date have been relegated to the case of targets in free space. There have been layered-medium results presented for the case of two-dimensional (2-D) scattering [22], as well as quasi-planar three-dimensional (3-D) problems in circuit and antenna design [23]. The difficulty of directly applying the FMM/MLFMA formalism to general 3-D layered-medium problems resides in the fact that the layered-medium Green's function is a dyadic, each term of which is represented in terms of a generally complicated Sommerfeld integral [8], [24]. One can use the complex-image technique [25]-[27] to represent each term of the dyadic in terms of a sum of images, generally located in complex space. Therefore, in principle, one can apply the fundamental FMM/MLFMA expansion to each term of this expansion. However, we have demonstrated [28], [29] that for the general case, the convergence (for complex image points) is often considerably slower than when source and observer are in real space. This mitigates the attractiveness of the underlying formalism.

In [28], [29], we therefore developed an approximate means of handling the dyadic half-space Green's function, with application to the FMM. In particular, the near FMM terms are evaluated via the use of the exact dyadic Green's function, the latter evaluated efficiently via the complex-image technique [25]-[27]. This part of the model is exactly as in a rigorous MoM analysis of scattering from targets in the vicinity of a half space [3], [8]. The far terms, evaluated efficiently via the clustering algorithm, employ the asymptotic form of the dyadic Green's function [30]. As elucidated further in 
the following, each component of the approximate Green's function is expressed in terms of the direct-radiation term plus radiation from an image source in real space [28]-[30]. The former accounts for the radiation of currents into the medium in which it resides, while the latter accounts for interactions with the half-space interface. The above approximation exploits the fact that the near interactions are most sensitive to accurate Green's-function evaluation, while the far interactions should be less so (the far interactions within the MLFMA formalism are always at least about half a wavelength apart [17]-[20]). Therefore, the half-space MLFMA presented here differs from the free-space MLFMA [17]-[20] in several ways. First, the nearby interactions are handled rigorously through use of the complete dyadic half-space Green's function, since these are likely to be most susceptible to approximations in the Green's function. For far interactions, we utilize the standard MLFMA clustering, with the interactions between such constituting the aforementioned direct radiation contribution. Finally, a separate set of image clusters, at real spatial positions, constitute the reflected component of the radiated fields. As is well known, the free-space FMM has $\mathrm{O}\left(N^{3 / 2}\right)$ memory and computational (CPU) requirements (per iteration) [14], [15], where $N$ is the number of unknowns for representation of the target, while the free-space MLFMA reduces these further to $\mathrm{O}(N \lg N)$ [17]-[20]. With the above construct, the real images introduce a new set of source clusters, and therefore, the algorithm requires slightly (typically about 20-60\%) more memory and about twice the computation time compared to the free-space version. However, the computational complexity of $\mathrm{O}(N \lg N)$, both in RAM and CPU (per iteration), remains unchanged.

The half-space FMM presented previously in [28], [29], which uses the aforementioned scheme for the efficient evaluation of the dyadic half-space Green's function, employed an electric-field integral equation, which limited its applicability somewhat, due to possible interior resonances for closed targets [9], [17]. In the work presented here, we therefore extend our previous work to an MLFMA formalism, employing electric-field (EFIE), magnetic-field (MFIE), and combined-field integral equations (CFIE), the latter eliminating interior resonances [9], [17].

The remainder of the paper is organized as follows. In Section II, we present our MLFMA formalism for surface or buried perfectly conducting targets. The model is validated in Section III-A through comparison with results from a rigorous body-ofrevolution MoM algorithm [1], [2]. In Section III-B and Section III-C, we present several results for realistic targets of interest to the remote sensing community. In particular, we consider scattering from large buried unexploded ordnance (UXO) and scattering from a realistic 3-D vehicle above ground. Memory and computational requirements for the half-space MoM, FMM, and MLFMA are compared. Finally, conclusions and suggestions for future work are discussed in Section IV.

\section{THEORY}

\section{A. Integral Equation and Half-Space MoM Formulation}

For solving the problem of scattering from an arbitrarily shaped 3-D, perfectly electric conducting (PEC) target situated

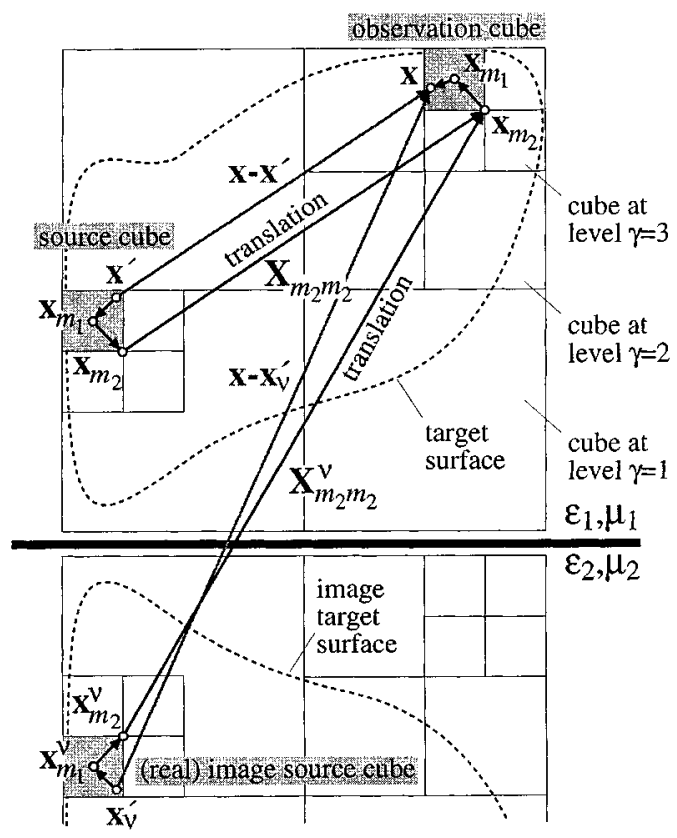

Fig. 1. Multilevel clustering in 3-D MLFMA and generalization to a half-space environment using real images to account for far interface interactions.

above (i.e., target in layer $i=1$ ) or buried (i.e., target in layer $i=2$ ) in a lossy half space (Fig. 1), we utilize the CFIE [9], [17]

$$
\begin{aligned}
\hat{\boldsymbol{t}} \cdot\left[\alpha \boldsymbol{E}^{i n c}(\boldsymbol{x})+\eta_{i}(1-\alpha) \hat{\boldsymbol{n}} \times \boldsymbol{H}^{i n c}(\boldsymbol{x})\right] \\
=\hat{\boldsymbol{t}} \cdot\left[-\alpha \boldsymbol{E}^{s c a t}(\boldsymbol{x})+\eta_{i}(1-\alpha) \boldsymbol{J}(\boldsymbol{x})-\eta_{i}(1-\alpha) \hat{\boldsymbol{n}}\right. \\
\left.\quad \times \boldsymbol{H}^{s c a t}\left(\boldsymbol{x} \in S^{+}\right)\right]
\end{aligned}
$$

with the scattered fields (inside half space $i$, in which the target is located) given by [8]

$$
\begin{aligned}
\boldsymbol{E}^{s c a t}(\boldsymbol{x})= & -j \omega \mu_{i}\left[\overleftrightarrow{\boldsymbol{I}}+\frac{\nabla \nabla}{k_{i}^{2}}\right] \cdot \iint_{S^{\prime}} \overleftrightarrow{G}_{A i i}\left(\boldsymbol{x}, \boldsymbol{x}^{\prime}\right) \cdot J\left(\boldsymbol{x}^{\prime}\right) d S^{\prime} \\
= & -j \omega \mu_{i} \iint_{S^{\prime}} \overleftrightarrow{K}_{A i i}\left(\boldsymbol{x}, \boldsymbol{x}^{\prime}\right) \cdot \boldsymbol{J}\left(\boldsymbol{x}^{\prime}\right) d S^{\prime} \\
& +\frac{\nabla}{j \omega \varepsilon_{i}} \iint_{S^{\prime}} K_{\phi e}^{i i}\left(\boldsymbol{x}, \boldsymbol{x}^{\prime}\right) \nabla^{\prime} \cdot J\left(\boldsymbol{x}^{\prime}\right) d S^{\prime} \\
\boldsymbol{H}^{\text {scat }}(\boldsymbol{x})= & \nabla \times \iint_{S^{\prime}} \overleftrightarrow{G}_{A i i}\left(\boldsymbol{x}, \boldsymbol{x}^{\prime}\right) \cdot J\left(\boldsymbol{x}^{\prime}\right) d S^{\prime}
\end{aligned}
$$

The unit vectors $\hat{\boldsymbol{n}}$ and $\hat{\boldsymbol{t}}$ are perpendicular and tangential to the scatterer surface, respectively, $\boldsymbol{x}$ is on and $\boldsymbol{x} \in S^{+}$is an infinitesimal distance outside the (closed) target surface. $\varepsilon_{i}=$ $\varepsilon_{i}^{\prime}-j \sigma_{i} / \omega, \mu_{i}$, and $k_{i}$ represent (in general complex) the permittivity, permeability, and wavenumber of the medium in which the target resides, and $\omega$ is the angular frequency (with a time dependence exp $j \omega t$ assumed and suppressed). Details on the Green's function dyadics $\overleftrightarrow{G}_{A i i}$ and $\overleftrightarrow{\boldsymbol{K}}_{A i i}$, as well as the scalar Green's function $K_{\phi e}^{i i}$ have been given by Michalski and Zheng in [8], where we use their formulation C. The CFIE in (1) is 
valid for a more general layered medium [8], but here we only consider the half-space problem for simplicity.

The CFIE (1) includes the electric-field integral equation (EFIE, $\alpha=1$ ) [9], which must be solved for an open target, and the magnetic-field integral equation (MFIE, $\alpha=0$ ) [9] as special cases. To avoid the possible spurious interior resonances of the EFIE and MFIE solutions [9], we typically choose $\alpha$ in the range of $\alpha=0.2-0.5$ if analyzing a closed target. It has been proven [9], [17], that the CFIE with $0<\alpha<1$ eliminates the effects of interior resonances.

In the conventional MoM solution, the unknown surface current $\boldsymbol{J}$ is expanded into a set of $N$ basis (expansion) functions $\boldsymbol{b}_{n^{\prime}}\left(\boldsymbol{x}^{\prime}\right)$

$$
\boldsymbol{J}\left(\boldsymbol{x}^{\prime}\right)=\sum_{n^{\prime}=1}^{N} I_{n^{\prime}} \boldsymbol{b}_{n^{\prime}}\left(\boldsymbol{x}^{\prime}\right)
$$

where we use the triangular patch Rao-Wilton-Glisson (RWG) basis introduced in [7]. Testing the CFIE (1) with a set of weighting (testing) functions $\boldsymbol{w}_{n}(\boldsymbol{x})$ tangential to the target surface results in $\mathrm{N}$ linear equations

$$
[Z] \cdot I=V
$$

for the unknown coefficients $I_{n^{\prime}}$. The elements of the driving vector $V$ (representing the incident field "tested" on the target surface) and the impedance matrix [ $Z$ ] are given by [14], [15], [17]

$$
V_{n}=\iint_{S} \boldsymbol{w}_{n}(\boldsymbol{x}) \cdot\left[\alpha \boldsymbol{E}^{i n c}(\boldsymbol{x})+\eta_{i}(1-\alpha) \hat{\boldsymbol{n}} \times \boldsymbol{H}^{i n c}(\boldsymbol{x})\right] d S
$$

and

$$
\begin{aligned}
Z_{n n^{\prime}}= & \alpha j \omega \mu_{i} \iint_{S} \iint_{S^{\prime}} \boldsymbol{w}_{n}(\boldsymbol{x}) \cdot\left[\overleftrightarrow{I}+\frac{\nabla \nabla}{k_{i}^{2}}\right] \\
& \cdot \overleftrightarrow{\boldsymbol{G}}_{A i i}\left(\boldsymbol{x}, \boldsymbol{x}^{\prime}\right) \cdot \boldsymbol{b}_{n^{\prime}}\left(\boldsymbol{x}^{\prime}\right) d S^{\prime} d S+\eta_{i}(1-\alpha) \iint_{S} \boldsymbol{w}_{n}(\boldsymbol{x}) \\
& \cdot\left[\begin{array}{l}
\boldsymbol{b}_{n^{\prime}}(\boldsymbol{x})-\hat{\boldsymbol{n}} \times \nabla \times \iint_{S^{\prime}} \overleftrightarrow{G}_{A i i}\left(\boldsymbol{x}, \boldsymbol{x}^{\prime}\right) \\
\left.\left.\cdot \boldsymbol{b}_{n^{\prime}}\left(\boldsymbol{x}^{\prime}\right) d S^{\prime}\right|_{\boldsymbol{x} \in S^{+}}\right] d S
\end{array}\right.
\end{aligned}
$$

respectively.

In an MLFMA analysis (Sections II-B and II-C), we divide the computation of interactions into near and far terms, where it must be emphasized that for far interactions, basis and weighting functions do not have to be in each other's far zone, but rather, these terms are handled by exploiting a plane wave spectral representation of the free-space Green's function (which requires that basis and weighting functions are "far enough" apart [14], [15], [19], but not necessarily in the far zone, see Section II-B). For near interactions (MoM part of the MLFMA), the evaluation of the impedance matrix elements (6), including the proper handling of self-term singularities and near singularity extraction, is done similar to the MoM literature [7], [31], [32]. The dyadic half-space Green's function is evaluated rigorously using the method of discrete complex images [25]-[27], thereby avoiding direct numerical evaluation of Sommerfeld integrals [8], [24]. Impedance matrix elements representing these near interactions are stored in a sparse matrix called [ $\left.Z^{\text {near }}\right]$. The method of complex images and explicit equations for the MoM impedance matrix elements can be found in the literature, and therefore, we do not repeat the details.

\section{B. Free-Space and Half-Space MLFMA}

The half-space dyadic Green's function can be split into a term $\overleftrightarrow{\boldsymbol{I}} g_{i}$ representing the "direct" radiation between source and observation point (as in free space, but using in general a complex wave number $k_{i}$ ) and a remaining dyadic $\Delta \overleftrightarrow{G}_{A i i}$ accounting for interactions with the interface (i.e., here $\Delta$ is not an operator) [8], [28], [29].

$$
\begin{aligned}
\overleftrightarrow{\boldsymbol{G}}_{A i i}\left(\boldsymbol{x}, \boldsymbol{x}^{\prime}\right) & =\overleftrightarrow{\boldsymbol{I}} g_{i}\left(\boldsymbol{x}, \boldsymbol{x}^{\prime}\right)+\Delta \overleftrightarrow{\boldsymbol{G}}_{A i i}\left(\boldsymbol{x}, \boldsymbol{x}^{\prime}\right) \\
& =\overleftrightarrow{\boldsymbol{I}} \frac{e^{-j k_{i}\left|\boldsymbol{x}-\boldsymbol{x}^{\prime}\right|}}{4 \pi\left|\boldsymbol{x}-\boldsymbol{x}^{\prime}\right|}+\Delta \overleftrightarrow{\boldsymbol{G}}_{A i i}\left(\boldsymbol{x}, \boldsymbol{x}^{\prime}\right)
\end{aligned}
$$

While the dyadic $\Delta \overleftrightarrow{G}_{A \ddot{i}}$ needs some further investigation (see below), the free-space FMM [14], [15] or MLFMA[17]-[20] can be applied to the "direct" term, with only minor changes due to the (in general) lossy background. The free-space FMM and MLFMA are based on the addition theorem [14], [21], leading to the (propagating) plane wave representation [14], [15], [21]

$$
\begin{aligned}
& g_{i}\left(\boldsymbol{x}, \boldsymbol{x}^{\prime}\right) \\
& =\frac{e^{-j k_{i}\left|\boldsymbol{x}-\boldsymbol{x}^{\prime}\right|}}{4 \pi\left|\boldsymbol{x}-\boldsymbol{x}^{\prime}\right|} \\
& \quad \approx \frac{-j k_{i}}{(4 \pi)^{2}} \iint_{4 \pi} e^{-j k_{i} \hat{\boldsymbol{k}} \cdot\left(\boldsymbol{x}-\boldsymbol{x}_{m}\right)} T_{L}\left(k_{i} X_{m^{\prime} m}, \hat{\boldsymbol{k}} \cdot \hat{\boldsymbol{X}}_{m^{\prime} m}\right) \\
& \quad \cdot e^{+j k_{i} \hat{\boldsymbol{k}} \cdot\left(\boldsymbol{x}^{\prime}-\boldsymbol{x}_{m^{\prime}}\right)} d^{2} \hat{\boldsymbol{k}} \\
& \quad=\sum_{l=0}^{L}(-j)^{l}(2 l+1) h_{l}^{(2)}\left(k_{i} X_{m^{\prime} m}\right) P_{l}\left(\hat{\boldsymbol{k}} \cdot \hat{\boldsymbol{X}}_{m^{\prime} m}\right) \\
& \left.\hat{\boldsymbol{X}}_{m^{\prime} m}, \hat{\boldsymbol{k}} \cdot \hat{\boldsymbol{X}}_{m^{\prime} m}\right) \\
& \quad \boldsymbol{X}_{m^{\prime} m} / X_{m^{\prime} m}=\boldsymbol{X}_{m^{\prime} m} / \sqrt{\boldsymbol{X}_{m^{\prime} m} \cdot \boldsymbol{X}_{m^{\prime} m}}
\end{aligned}
$$

of the scalar Green's function. The distance vector $\boldsymbol{R}=\boldsymbol{x}-\boldsymbol{x}^{\prime}$ has been subdivided into a vector from the source point $\boldsymbol{x}^{\prime}$ to the center $\boldsymbol{x}_{m^{\prime}}$ of a "source group," a vector from an "observation group" center $\boldsymbol{x}_{m}$ to the observation point $\boldsymbol{x}$, and a vector $\boldsymbol{X}_{m^{\prime} m}$ connecting the group centers (Fig. 1, where in this Section II-B, we write $m^{\prime}=m_{\gamma}^{\prime}, m=m_{\gamma}$, and $\boldsymbol{X}_{m^{\prime} m}=\boldsymbol{X}_{m_{\gamma}^{\prime} m_{\gamma}}$ for simplification)

$$
\boldsymbol{R}=\boldsymbol{x}-\boldsymbol{x}^{\prime}=\left(\boldsymbol{x}-\boldsymbol{x}_{m}\right)+\boldsymbol{X}_{m^{\prime} m}-\left(\boldsymbol{x}^{\prime}-\boldsymbol{x}_{m^{\prime}}\right)=\boldsymbol{X}_{m^{\prime} m}+\boldsymbol{d} .
$$

Details on the convergence of the addition theorem [14], [21], resulting in approximate formulas for the minimum number $L$ 
of terms needed for a desired accuracy, can be found in the literature [14], [15], [19] for a real wavenumber $k_{i}=k_{0}$. In the case of a lossy background (e.g., soil) more terms are required [29]. Therefore, in our implementation, $L$ is determined adaptively for a predefined minimum separation of the group centers, the latter typically chosen in the range $1.5 d_{\max }-2 d_{\max }$, where $d_{\max }$ is the maximum group diameter. For groups situated closer together, the interactions are included in the sparse matrix $\left[Z^{\text {near }}\right]$ (Section II-A). A more detailed discussion was given in [29]. We should also mention that for all examples in Section III, the upper limit $L$ in the series expansion has been determined to provide an error of less than $1 \%$ in the underlying addition theorem [14], [21]. For the efficient numerical evaluation of (8a) over the solid angle $4 \pi$, a Gaussian quadrature integration with $K=2 L^{2}$ plane wave directions $\hat{\boldsymbol{k}}$ is applied [14], [15], although more efficient quadrature rules are available [33].

To incorporate the expansion (8) into a fast algorithm for the iterative solution of the matrix equation (4), the scatterer surface has to be partitioned first into groups. While in the single-stage FMM, one level of $M \sim \sqrt{N}$ groups is applied [14], [15], the MLFMA firstencloses the objectinalarge cube of edgelength $d$, and then the cube (or subcube) is recursively divided into eight smaller cubes until the edge length $d / 2^{g}$ at the finest level $\gamma=g$ is approximately half a wavelength [17]-[20] (Fig. 1). Only information for thenonempty cubesis stored using tree-structured data. Therefore, the memory and computational costs depend on nonempty cubes only [17]-[20]. In the following, basis/weighting functions numbered globally as $n(m, \alpha)=1 \cdots N$ are also labeled according to a group (cube) index $m=1 \cdots M$ and an additional index $\alpha=1 \cdots A_{m}$ within each group (cube). For a specific MLFMA level $\gamma$, these variables will be denoted as $m_{\gamma}, M_{\gamma}, \alpha_{\gamma}$, and $A_{m_{\gamma}}$, respectively.

Using the expansion (8), the elements of the far interaction impedance matrix (i.e., for $X_{m^{\prime} m}$ sufficiently large) in the context of a free-space scattering problem can be written as [17]

$$
\begin{aligned}
Z_{n n^{\prime}} \approx & \frac{\omega \mu_{i} k_{i}}{(4 \pi)^{2}} \iint_{4 \pi} \boldsymbol{W}_{m \alpha}(\hat{\boldsymbol{k}}) \cdot T_{L}\left(k_{i} X_{m^{\prime} m}, \hat{\boldsymbol{k}} \cdot \hat{\boldsymbol{X}}_{m^{\prime} m}\right) \\
& \cdot \boldsymbol{B}_{m^{\prime} \alpha^{\prime}}(\hat{\boldsymbol{k}}) d^{2} \hat{\boldsymbol{k}} \\
\boldsymbol{B}_{m^{\prime} \alpha^{\prime}}(\hat{\boldsymbol{k}})= & {[\overleftrightarrow{\boldsymbol{I}}-\hat{\boldsymbol{k}} \hat{\boldsymbol{k}}] \cdot \iint_{S^{\prime}} \boldsymbol{b}_{n^{\prime}\left(m^{\prime}, \alpha^{\prime}\right)}\left(\boldsymbol{x}^{\prime}\right) } \\
\boldsymbol{W}_{m \alpha}(\hat{\boldsymbol{k}})= & \alpha[\overleftrightarrow{\boldsymbol{I}}-\hat{\boldsymbol{k}} \hat{\boldsymbol{k}}] \cdot \iint_{S} \boldsymbol{w}_{n(m, \alpha)}(\boldsymbol{x}) \\
& \cdot e^{-j k_{i} \hat{\boldsymbol{k}} \cdot\left(\boldsymbol{x}-\boldsymbol{x}_{m}^{\prime}-\boldsymbol{x}_{m^{\prime}}\right)} d S^{\prime} \\
& +(1-\alpha) \hat{\boldsymbol{k}} \times \iint_{S} \hat{\boldsymbol{n}} \times \boldsymbol{w}_{n(m, \alpha)}(\boldsymbol{x}) \\
& \cdot e^{-j k_{i} \hat{\boldsymbol{k}} \cdot\left(\boldsymbol{x}-\boldsymbol{x}_{m}\right)}, d S
\end{aligned}
$$

where (11) and (12) represent the far-field radiation pattern of the basis functions and weighting pattern of the testing functions, respectively. Thus, the expansion (8) is first used to translate each plane wave component of the field radiated by the in- dividual basis functions within a source group into a common center $\boldsymbol{x}_{m^{\prime}}$ (aggregation). The spectral components $\boldsymbol{B}_{m^{\prime} \alpha^{\prime}}(\hat{\boldsymbol{k}})$ are then shifted individually to the common center $\boldsymbol{x}_{m}$ of a group of weighting functions using the operators $T_{L}$ (translation). Finally, the plane waves are weighted according to the receiving patterns $\boldsymbol{W}_{m \alpha}(\hat{\boldsymbol{k}})$, including the redistribution of the fields from the common center to the location of the weighting functions (disaggregation).

For a half-space FMM/MLFMA, it is essential to include the effects of the far interface interactions. In the complex-image technique, each component of the dyadic $\Delta \overleftrightarrow{\boldsymbol{G}}_{A i i}$ is expressed in terms of a sum of free-space Green's functions with image sources located in complex space [25]-[27]. Therefore, in principle, the expansion (8) (which remains valid for general complex source points [21]) could also be applied for far interface interactions. However, we have shown recently [28], [29], that the number of terms L required for convergence can be prohibitively large for general complex source points, undermining the efficiency of using (8) for far interface interactions in the context of the discrete complex-image technique.

In [28] and [29] we therefore described an alternative (though approximate) formulation. While the half-space dyadic Green's function is rigorously accounted for in the near interaction matrix, far interactions are often less sensitive to approximations in the Green's function [28]-[30]. The FMM has been successfully extended to the scattering from a PEC object above or buried in a half space by employing the asymptotic form of the Green's function [30] for far interactions. The asymptotic form of the Green's function is represented utilizing a single real image at $[\overleftrightarrow{\boldsymbol{I}}-2 \hat{z} \hat{\boldsymbol{z}}] \cdot \boldsymbol{x}^{\prime}$ (assuming the interface at $z=0$ ) with its polarization dependent magnitude given by the reflection dyadic [30]

$$
\begin{gathered}
\overleftrightarrow{\boldsymbol{r}}(\hat{\boldsymbol{k}})=\hat{\boldsymbol{h}} \hat{\boldsymbol{h}} R_{\mathrm{TE}}(\hat{\boldsymbol{k}})+[\overleftrightarrow{\boldsymbol{I}}-\hat{\boldsymbol{h}} \hat{\boldsymbol{h}}] R_{\mathrm{TM}}(\hat{\boldsymbol{k}}) \\
\text { with } \hat{\boldsymbol{h}}=\frac{\hat{z} \times \hat{\boldsymbol{k}}}{|\hat{z} \times \hat{\boldsymbol{k}}|} .
\end{gathered}
$$

Therefore, in addition to the accurate calculation of the halfspace dyadic Green's function in the near interaction matrix [ $Z^{\text {near }}$ ] (Section II-A), the half-space MLFMA only requires the definition of a single set of real image sources (Fig. 1), which can be handled similarly to real sources. Generalizing the free-space MLFMA [17]-[19] to a half-space MLFMA is now straightforward. Besides some additional operations in the matrix-vector product (Section II-C), the preprocessing stage has to include calculations of the translation operators

$$
\begin{aligned}
& T_{L}\left(k_{i} X_{m^{\prime} m}^{\nu}, \hat{\boldsymbol{k}} \cdot \hat{\boldsymbol{X}}_{m^{\prime} m}^{\nu}\right) \\
& \quad=\sum_{l=0}^{L}(-j)^{l}(2 l+1) h_{l}^{(2)}\left(k_{i} X_{m^{\prime} m}^{\nu}\right) P_{l}\left(\hat{\boldsymbol{k}} \cdot \hat{\boldsymbol{X}}_{m^{\prime} m}^{\nu}\right)
\end{aligned}
$$

between image cube and observation cube centers for all nonnearby cubes at all levels, as well as the Fourier transforms

$$
\begin{aligned}
\boldsymbol{B}_{m^{\prime} \alpha^{\prime}}^{\nu}(\hat{\boldsymbol{k}})= & {[\overleftrightarrow{\boldsymbol{I}}-\hat{\boldsymbol{k}} \hat{\boldsymbol{k}}] \cdot[\overleftrightarrow{\boldsymbol{I}}-2 \hat{z} \hat{z}] \cdot \iint_{S^{\prime}} \boldsymbol{b}_{n^{\prime}\left(m^{\prime}, \alpha^{\prime}\right)}\left(\hat{\boldsymbol{x}}^{\prime}\right) } \\
& \cdot e^{+j k_{i} \hat{\boldsymbol{k}} \cdot[\overleftrightarrow{\boldsymbol{I}}-2 \hat{z} \hat{z}] \cdot\left(\boldsymbol{x}^{\prime}-\hat{\boldsymbol{x}}_{m^{\prime}}\right)} d S^{\prime}
\end{aligned}
$$


of the image expansion functions $[\overleftrightarrow{\boldsymbol{I}}-2 \hat{\boldsymbol{z}} \hat{z}] \cdot \boldsymbol{b}_{n^{\prime}}\left(\hat{\boldsymbol{x}}^{\prime}\right)$, where $\boldsymbol{X}_{m^{\prime} m}^{\nu}$ is a vector from the image source group center $\boldsymbol{x}_{m^{\prime}}^{\nu}$ to the observation group center (Fig. 1), and the dyadic $\overleftrightarrow{I}-2 \hat{z} \hat{z}$ accounts for the relative orientation and location of the image expansion functions, respectively.

Before proceeding, we should add a few comments on the image sources and their respective clustering. One might argue that we could include all sources (i.e., sources and real image sources) in a single big box, to which we could then apply the conventional free-space MLFMA (with general complex wavenumber). However, each image magnitude depends on the plane-wave direction (or, equivalently, on the relative location of source and observer). This is in contrast to the behavior of the sources in a free-space MLFMA. Another interesting issue arises from a comparison of the outgoing plane wave expansions in (11) and (15). Some reductions in computational complexity and memory are possible when computing and storing only one set of vectors, although this approach has not been included in our numerical implementation up to now.

\section{Matrix-Vector Product in Half-Space MLFMA}

The MLFMA accelerates the solution of the integral equation (1) by reducing the complexity of the required RAM and the CPU needed for a single matrix-vector multiplication in an iterative conjugate gradient solution of (4) [34], [35] from $\mathrm{O}\left(N^{2}\right)$ for the MoM to $\mathrm{O}(N \lg N)$ for the MLFMA [17]-[20]. We investigated several iterative solvers, including the standard conjugate gradient method operating on the normal equation (CGN), the biconjugate gradient (BiCG), the biconjugate gradient stabilized (BiCGStab), the conjugate gradient squared (CGS), and the transpose free quasi-minimum residual (TFQMR) methods [34], [35]. The latter three have the advantage that they only perform matrix-vector products $[Z] \cdot I$, while the $\mathrm{CGN}$ and $\mathrm{BiCG}$ methods also require the product $[\boldsymbol{Z}]^{\dagger} \cdot \boldsymbol{I}$ with the adjoint (i.e., conjugate transpose) matrix. Interestingly, for the problems we investigated up to now, the CGN method was often the best choice (see [36]), at least without a preconditioner. However, while the reduction in the number of iterations in the CGN when using a block-diagonal preconditioning scheme [19], [35] was moderate, we observed a significant speedup in the BiCGStab, CGS, and TFQMR, typically reducing the number of iterations by a factor of two to four.

Following the discussion in [17] for the free-space MLFMA,we briefly summarize the basic steps for performing the matrix-vector product $\boldsymbol{y}=[Z] \cdot \boldsymbol{I}$ in the half-space MLFMA (the adjoint-vector product $[\boldsymbol{Z}]^{\dagger} \cdot \boldsymbol{I}$ can be derived in a similar way, if needed in the iterative solver). It consists of two sweeps and a final weighting by the plane wave expansion of the testing functions.

For a given vector $\boldsymbol{I}$, the first sweep calculates the outgoing plane wave expansions (i.e., the radiation patterns) of all nonempty cubes at all levels $\gamma=1 \cdots g$, where the number $K_{\gamma}=2 L_{\gamma}^{2}$ of plane waves necessarily increases with increasing cube dimensions [14], [15], [17], [19] (i.e., it increases when going from the finest level $\gamma=g$ to the coarsest level $\gamma=$ 1). While the outgoing plane wave expansions at the finest level $g$ are calculated directly using the radiation patterns
$\boldsymbol{B}_{m^{\prime} \alpha^{\prime}}(\hat{\boldsymbol{k}})$ and $\boldsymbol{B}_{m^{\prime} \alpha^{\prime}}^{\nu}(\hat{\boldsymbol{k}})$ of the basis and image basis function, respectively

$$
\begin{array}{r}
\boldsymbol{s}_{m_{g}^{\prime}}^{(\nu)}\left(\hat{\boldsymbol{k}}_{\kappa_{g}}\right)=\sum_{\alpha_{g}^{\prime}=1}^{A_{m_{g}^{\prime}}} I_{n^{\prime}\left(m_{g}^{\prime}, \alpha_{g}^{\prime}\right)} \boldsymbol{B}_{m_{g}^{\prime} \alpha_{g}^{\prime}}^{(\nu)}\left(\hat{\boldsymbol{k}}_{\kappa_{g}}\right) \\
\text { for }\left\{\begin{array}{c}
m_{g}^{\prime}=1,2, \cdots, M_{g} \\
\kappa_{g}=1,2, \cdots, K_{g}
\end{array}\right.
\end{array}
$$

the plane wave expansions at all remaining levels are obtained using shifting and interpolation [16], [17]. The interpolation step is necessary because the required number of plane waves $K_{\gamma}$ at the coarser level $\gamma$ is larger than the number of plane waves $K_{\gamma+1}$ at level $\gamma+1$. Therefore, the expansions $\boldsymbol{s}_{m^{\prime}}^{(\nu)}$ are first interpolated (using a sparse interpolation matrix $\left[W^{+1}\right]$ according to a third-order Lagrange interpolation) and are then shifted to the cube center at the coarser level $\gamma$, and finally, the contribution from all child cubes is added

$$
\begin{aligned}
\boldsymbol{s}_{m_{\gamma}^{\prime}}^{(\nu)}\left(\hat{\boldsymbol{k}}_{\kappa_{\gamma}}\right)= & \sum_{\substack{\text { child } \\
\text { cubes }}} e^{-j k_{i} \hat{\boldsymbol{k}}_{\kappa \gamma} \cdot\left(\boldsymbol{x}_{m_{\gamma}^{\prime}}-\hat{\boldsymbol{x}}_{m_{\gamma+1}^{\prime}}\right)} \\
& \cdot \sum_{\kappa_{\gamma+1}=1}^{K_{\gamma+1}} W_{\kappa_{\gamma} \kappa_{\gamma+1} \boldsymbol{s}_{m_{\gamma+1}^{\prime}}^{(\nu)}\left(\hat{\boldsymbol{k}}_{\kappa_{\gamma+1}}\right)} \\
& \text { for }\left\{\begin{array}{l}
\gamma=g-1, \cdots, 2,1 \\
m_{\gamma}^{\prime}=1,2, \cdots, M_{\gamma} . \\
\kappa_{\gamma}=1,2, \cdots, K_{\gamma}
\end{array}\right.
\end{aligned}
$$

The second sweep calculates the incoming plane wave expansions at the finest level $\gamma=g$, starting with a direct calculation at the coarsest level $\gamma=1$ and then recursively going to finer levels using shifting and anterpolation [16], [17]. At the coarsest level, the incoming plane wave expansions with phase reference at the cube centers are given by

$$
\begin{aligned}
& \boldsymbol{g}_{m_{1}}\left(\hat{\boldsymbol{k}}_{\kappa_{1}}\right)=
\end{aligned}
$$

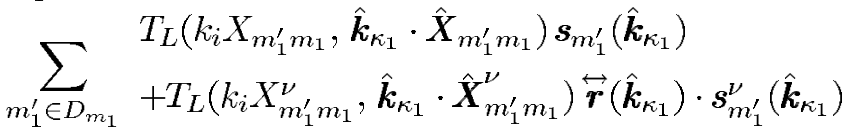

$$
\begin{aligned}
& \text { for }\left\{\begin{array}{c}
m_{1}=1,2, \cdots, M_{1} \\
\kappa_{1}=1,2, \cdots, K_{1}
\end{array}\right. \\
& \boldsymbol{g}_{m_{\gamma}}\left(\hat{\boldsymbol{k}}_{\boldsymbol{\kappa}_{\gamma}}\right)=
\end{aligned}
$$

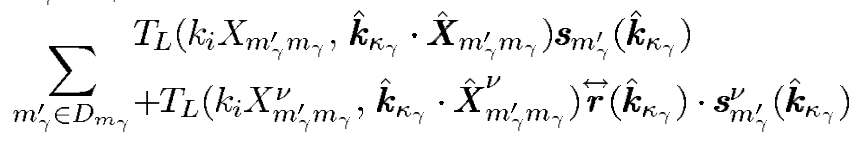

$$
\begin{aligned}
& +\sum_{\kappa_{\gamma-1}=1}^{K_{\gamma-1}} \frac{w_{\kappa_{\gamma-1}}}{w_{\kappa_{\gamma}}} W_{\kappa_{\gamma-1} \kappa_{\gamma}} g_{m_{\gamma-1}}\left(\hat{\boldsymbol{k}}_{\kappa_{\gamma-1}}\right) \\
& \cdot e^{+j k_{i} \hat{\boldsymbol{k}}_{\kappa_{\gamma-1}} \cdot\left(\boldsymbol{x}_{m_{\gamma-1}}-\boldsymbol{x}_{m_{\gamma}}\right)} \\
& \text { for }\left\{\begin{array}{c}
m_{\gamma}=1,2, \cdots, M_{\gamma} \\
\kappa_{\gamma}=1,2, \cdots, K_{\gamma}
\end{array}\right.
\end{aligned}
$$

where $D_{m_{\gamma}}$ denotes all cubes well-separated (far) from cube $m_{\gamma}$ at level $\gamma$ (but not well-separated at the parent level $\gamma-1$ ). The incoming plane wave expansions at successively finer levels $\gamma$ are given by a contribution from all well-separated cubes at this level (but not well-separated at the parent level) and the 
contribution from well-separated cubes at the parent level, the latter calculated using shifting and anterpolation as described in [16], [17], where the factors $w_{\kappa_{\gamma}}$ are the weighting coefficients of the $K_{\gamma}=2 L_{\gamma}^{2}$-point Gaussian quadrature integration applied at level $\gamma$.

Finally, the resulting vector $\boldsymbol{y}=[\boldsymbol{Z}] \cdot \boldsymbol{I}$ of the matrix-vector multiplication is given by

$$
\begin{aligned}
y_{n}= & y_{n\left(m_{g}, \alpha_{g}\right)} \\
= & \sum_{n^{\prime}=1}^{N} Z_{n n^{\prime}}^{\text {near }} I_{n^{\prime}}+\frac{\omega \mu_{i} k_{i}}{(4 \pi)^{2}} \iint_{4 \pi} \boldsymbol{W}_{m_{g} \alpha_{g}}(\hat{\boldsymbol{k}}) \cdot \boldsymbol{g}_{m_{g}}(\hat{\boldsymbol{k}}) d^{2} \hat{\boldsymbol{k}} \\
\approx & \sum_{n^{\prime}=1}^{N} Z_{n n^{\prime}}^{\text {near }} I_{n^{\prime}}+\frac{\omega \mu_{i} k_{i}}{(4 \pi)^{2}} \sum_{\kappa_{g}=1}^{K_{g}} w_{\kappa_{g}} \boldsymbol{W}_{m_{g} \alpha_{g}}\left(\hat{\boldsymbol{k}}_{\kappa_{g}}\right) \\
& \cdot \boldsymbol{g}_{m_{g}}\left(\hat{\boldsymbol{k}}_{\kappa_{g}}\right) \quad \text { for } n=1,2, \cdots, N
\end{aligned}
$$

where the first term accounts for near interactions (utilizing the sparse matrix [ $Z^{\text {near }}$ ] introduced in Section II-A). Far interactions, i.e., the second term in (18), are included by an appropriate weighting (applying the receiving pattern of the weighting function and a Gaussian quadrature integration) of the incoming plane wave expansion with phase reference at the center $\boldsymbol{x}_{m_{g}}$ of the cube in which the weighting function $\boldsymbol{w}_{n}$ is located.

As can be easily seen, the first and second sweep require approximately twice the number of operations compared to a free-space MLFMA, and the final (and computationally less intensive) step (18) is unchanged. Therefore, the number of operations needed for one matrix-vector product only increases by a factor of about two compared to the free-space MLFMA. While the same is true for the calculation of the translation operators in the preprocessing stage (Section II-B), the increase in CPU for calculating the Fourier transforms of basis and weighting functions is even smaller (only about $50 \%$ ). There is only one additional set of Fourier transforms (15) for the image basis functions but none for the weighting functions. Only the number of operations required for the evaluation of the sparse matrix [ $\left.Z^{\text {near }}\right]$ does (in general) not scale with a factor of two (or less) compared to the free-space MLFMA, depending on the number of complex images needed for an accurate representation of the half-space dyadic Green's function. Nevertheless, for large scattering problems the CPU time for the iterative solution often dominates, and therefore an overall increase in the CPU time on the order of two was observed in most of our calculations relative to the free-space case. Before leaving the theoretical part of this paper, it should be mentioned also that the increase in RAM is always less than $100 \%$ (typically on the order of 20-60\%) compared to the free-space case. An additional set of translation operators (14) and plane wave expansions of the image basis functions (15) must be stored in memory, but the memory required for the sparse matrix [ $Z^{\text {near }}$ ] is unchanged. Therefore, the overall complexity in RAM as well as CPU (for one step in the iterative solver) remains $\mathrm{O}(N \lg N)$ like in the free-space MLFMA [17]-[20].

\section{RESULTS}

All results are presented for perfectly conducting (PEC) targets in the presence of a half space, with the electrical properties

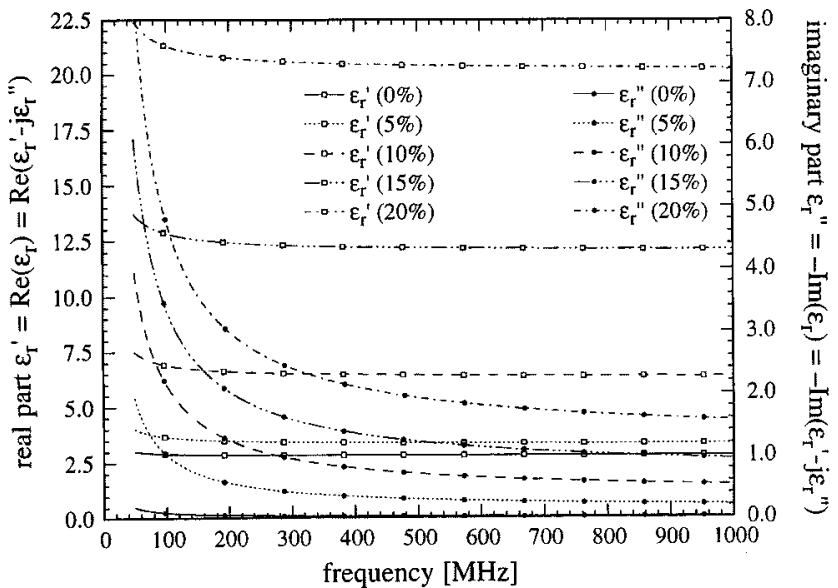

Fig. 2. Frequency dependent permittivity (real and imaginary part) of Yuma soil for different water content (percentage by weight) [37].

of the soil representative of soil samples taken from Yuma, AZ [37]. The soil electrical properties over the frequency range of interest are shown in Fig. 2 as a function of water content by percentage weight. We consider plane-wave excitation and all scattered fields are computed in the far zone. Results are shown for three target types, each of interest to remote sensing. First, we consider scattering from a perfectly conducting cylinder over a half space, with the cylinder axis normal to the half-space interface. Such rotationally symmetric targets, which give rise to no cross-polarized backscattered fields [38], are convenient calibration targets. The accuracy of the MLFMA for this target is verified through comparison with results computed via a MoM algorithm specialized to the case of a body of revolution (BOR). We next consider scattering from a buried missile-like target, of interest for the detection of buried UXO. In that example, we compare the MLFMA results to MoM and FMM solutions. Finally, we consider scattering from a vehicle-like target, of interest for military remote sensing. Due to the large size of that target, we do not have separate MoM results for comparison, although the previous two examples are meant to validate the accuracy of the MLFMA. We have found that (for closed targets) conjugate-gradient convergence is significantly improved by use of a CFIE formulation, vis-à-vis an EFIE or MFIE-based solution. This is in addition to the other salutary properties of a CFIE (no interior resonances [9], [17]). Therefore, all MLFMA computations employ the CFIE formulation.

\section{A. Cylinder Over a Half Space}

Consider a PEC cylinder situated over a half space, as shown inset in Fig. 3. The cylinder is $3 \mathrm{~m}$ long, has a diameter of $1 \mathrm{~m}$, and is situated $20 \mathrm{~cm}$ over the soil interface. Electromagnetic scattering from this target can be analyzed via a specialized MoM solution tailored to a BOR [1], [2]. The BOR-MoM provides a good model for comparison to the MLFMA, because the BOR basis functions are entirely different than the RWG triangular patch basis functions [7] employed in the MLFMA. In particular, the BOR solution exploits the rotational symmetry of the target-(half space) composite, expanding both the Green's function and surface currents in a Fourier-series representation. 


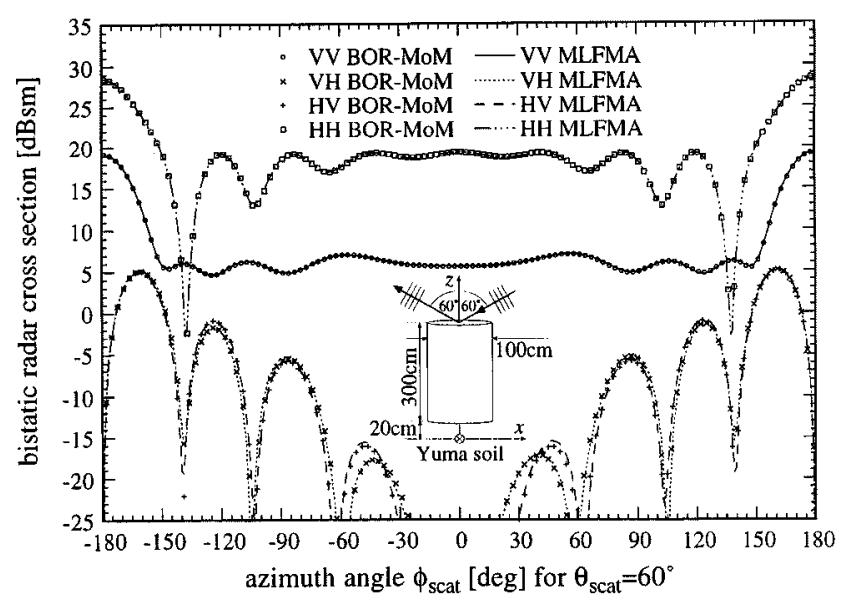

Fig. 3. RCS of a conducting cylinder of height $300 \mathrm{~cm}$ and diameter $100 \mathrm{~cm}$ (see inset and mesh in Fig. 4) situated $20 \mathrm{~cm}$ above Yuma soil of $10 \%$ water content (see Fig. 2). A plane wave is incident from $\theta_{i n c}=60^{\circ}$ (i.e., $30^{\circ}$ from grazing) and $\phi_{i n c}=0^{\circ}$. The figure shows the bistatic RCS for $\theta_{\text {scat }}=60^{\circ}$ and a frequency of $f=600 \mathrm{MHz}$. The MLFMA results (CFIE with four levels, $N=15519$ unknowns, $548 \mathrm{MB}$ of RAM) are compared to the RCS values computed via a rigorous BOR MoM (EFIE) [1], [2].

Consequently, the BOR solution is characterized by a current expansion along the generating arc only, vis-à-vis the MLFMA, which employs 3-D triangular patches to cover the whole target surface. The MLFMA basis functions are expanded at a rate of about 10 per wavelength, while for the purposes of providing a benchmark reference solution, the BOR basis functions are expanded at a rate of 20 subsectional basis functions per wavelength along the generating arc. In these examples the BOR-MoM results employ an EFIE [1], [8], while the MLFMA employs a CFIE.

In Fig. 3, we consider operation at a frequency of $600 \mathrm{MHz}$, which implies that the target is six wavelengths long, constituting a relatively large target, electrically. In particular, the MLFMA solution is characterized by $N=15519$ unknowns (548 MB of RAM), for which we employ a four-level solution. The incident plane wave direction is fixed at $\phi_{\text {inc }}=0^{\circ}$ and $\theta_{\text {inc }}=60^{\circ}$, and the bistatic RCS is observed at $\theta_{\text {scat }}=60^{\circ}$ for all $\phi_{\text {scat }}$. Note that at $\phi_{\text {scat }}=0^{\circ}$, the results correspond to backscatter, for which the cross-polarized fields vanish for this rotationally symmetric target [38]. In addition to noting the very close agreement between the BOR-MoM and MLFMA solutions, several other interesting observations can be gleaned from these results. In particular, the results presented here are for soil properties characteristic of Yuma soil with $10 \%$ water content (see Fig. 2). For such, the Brewster angle corresponds to approximately $68.8^{\circ}$ (for lossy soil, there is no exact Brewster angle). The plane-wave incident angle considered here is close enough to $68.8^{\circ}$ that very good soil penetration (and therefore little reflection) is achieved for vertical polarization. This explains why the $\mathrm{HH}$ response is considerably larger than its VV counterpart, the dihedral response of the latter being reduced significantly by soil penetration.

Before proceeding, we consider the cross-polarization results in Fig. 3. As expected, the cross-polarized fields are considerably smaller than their copolarized counterparts. An interesting issue involves reciprocity, from which we expect the $\mathrm{VH}$ and HV scattered fields to be identical. This is due to the specific incident and (bistatic) scattering directions used in this example, even though this is not true for the general bistatic case. Above, approximately $-15 \mathrm{dBsm}$ (about $45 \mathrm{~dB}$ below the maximum copolarized RCS), reciprocity is satisfied well. However, below $-15 \mathrm{dBsm}$ there is a noticeable difference between the VH and HV scattered fields. Interestingly, this does not appear to be a problem associated specifically with the MLFMA, since it is in very good agreement with the BOR-MoM results for both cross-polarized fields considered. We therefore attribute the errors in satisfying reciprocity to inaccuracies in computing the Green's function. The BOR-MoM model employs the method of complex images [25]-[27] for all (expansion function)-(testing function) interactions, while the MLFMA uses this same technique for the near interactions (Section II-A). In the complex-image technique, we fit each individual dyadic component of the spectral-domain Green's function to an exponential expansion with complex arguments, with the fit achieved via a least-square Prony's method [39]. We therefore set a predefined acceptable relative error between the exact and Prony representation of the spectral Green's function, with that error set to $1 \%$ in all our computations. Small errors in the expansion give rise to the errors in Fig. 3, manifested in the cross-polarized RCS. Nevertheless, the results demonstrate a dynamic range of approximately $45 \mathrm{~dB}$, with the significant cross-polarization errors occurring primarily at angles for which the VH and HV RCS are very small.

We note that for a sampling rate of 20 basis functions per wavelength along the generating arc, the BOR-MoM results reported in Fig. 3 required $101 \mathrm{MB}$ of RAM and about 70 min of CPU time (these numbers reduce to $26 \mathrm{MB}$ and approximately 18 min for ten expansion functions per wavelength). By comparison, the MLFMA required $548 \mathrm{MB}$ and nearly $90 \mathrm{~min}$ of CPU, at a sampling rate of ten basis functions per wavelength, with both the BOR-MoM and MLFMA computations performed on a SGI R10000/195 MHz processor. This raises an interesting question: For specialized targets such as the BOR in Fig. 3, when is it better to treat scattering with a specialized BOR-MoM code versus a more general but also highly efficient MLFMA scheme? In the BOR-MoM computations of Fig. 3, azimuthal modes $e^{ \pm j m \phi}$ with $m$ ranging from $m=0$ to $m=12$ were employed, based on the empirical relation in [2]. As the frequency increases, the number of azimuthal modes required increases, and the BOR-MoM formalism becomes less efficient. Numerical experiments have revealed that when the frequency of operation is increased above $1 \mathrm{GHz}$ (see Fig. 4), the MLFMA becomes faster than the BOR-MoM solution. Such issues are addressed in greater detail in the future.

The results in Fig. 3 considered bistatic scattering at a single frequency. To address the performance of the MLFMA as a function of frequency, in Fig. 4 we present backscattered RCS for the incident angles considered in Fig. 3. The MLFMA and BOR-MoM employed a sampling of approximately ten basis functions per wavelength (recall that the MLFMA and BOR-MoM solutions are characterized by distinct basis-function types). As above, the BOR-MoM employs an EFIE, while the MLFMA is based on a CFIE formulation. We see in Fig. 4 


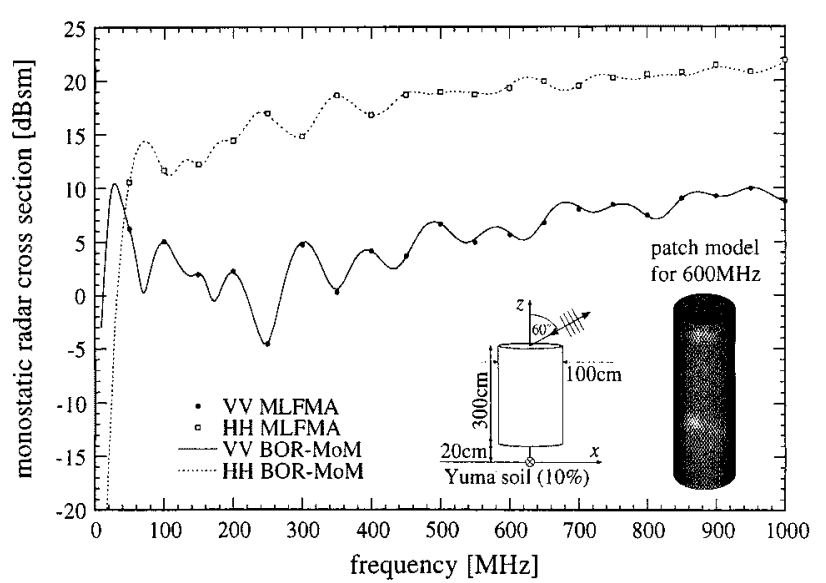

Fig. 4. As in Fig. 3, but plotting the monostatic RCS as a function of frequency. The discretization was fixed to about $\lambda 10$, for both the MLFMA and the body-of-revolution MoM. At $f=1000 \mathrm{MHz}$ the MLFMA used $N=$ 42873 unknowns, five levels, and a total of $1.57 \mathrm{~GB}$ of RAM.

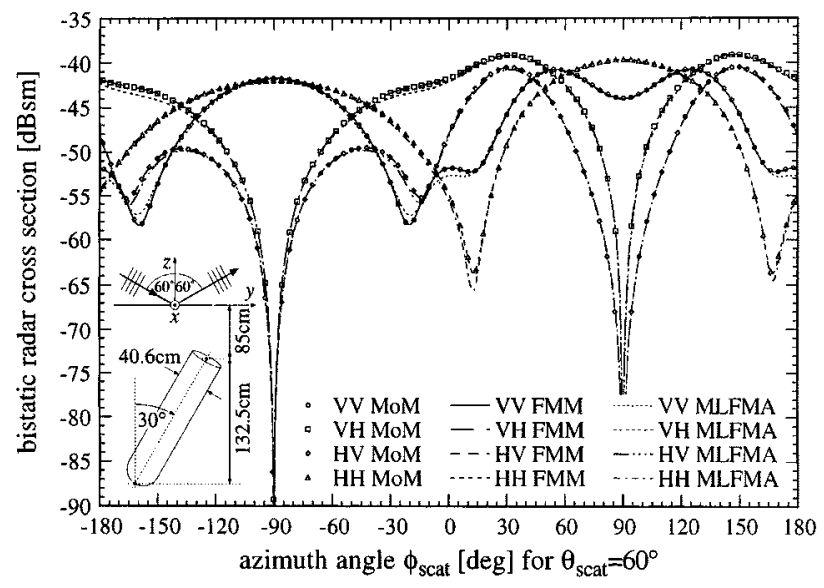

Fig. 5. Bistatic RCS of a model UXO (cylinder with hemispherical endcap) of length $153 \mathrm{~cm}$ and diameter $40.6 \mathrm{~cm}$ (see inset). The model UXO is buried in Yuma soil of 5\% water content (see Fig. 2) with the target symmetry axis in the $y z$-plane, at an angle of $30^{\circ}$ relative to the $z$-axis and the nose down at a depth $z=-217.5 \mathrm{~cm}$. The RCS is plotted for varying $\phi_{\text {scat }}$ at a fixed angle $\theta_{\text {scat }}=60^{\circ}$ and a frequency of $600 \mathrm{MHz}(N=10653$ unknowns, see mesh in Fig. 6), assuming a plane wave incident at $\phi_{i n c}=-90^{\circ}$ (in the $y z$-plane) and $\theta_{\text {inc }}=60^{\circ}$. Results from an MoM (using EFIE), FMM (using EFIE), and MLFMA (using CFIE and four levels) code are compared.

that the agreement between the MLFMA and BOR-MoM is excellent, for all frequencies considered. Results are only shown for copolarization, the cross-polarized fields vanishing in backscatter, as discussed above. To get a feel for the complexity, at $300 \mathrm{MHz}$ the MLFMA required $161 \mathrm{MB}$ of RAM and $20 \mathrm{~min}$ of CPU (3936 unknowns, three levels), while at $1 \mathrm{GHz}$, these numbers are $1.57 \mathrm{~GB}$ and $227 \mathrm{~min}(42873$ unknowns, five levels), where all computations were run on a single SGI R10000/195 MHz processor.

\section{B. Buried Unexploded Ordnance}

In our next example we consider the ordnance-like target inset in Fig. 5, with the soil characterized by 5\% water content (see Fig. 2). We compare results from the MLFMA, computed with a four-level CFIE, to results computed by the MoM and FMM,

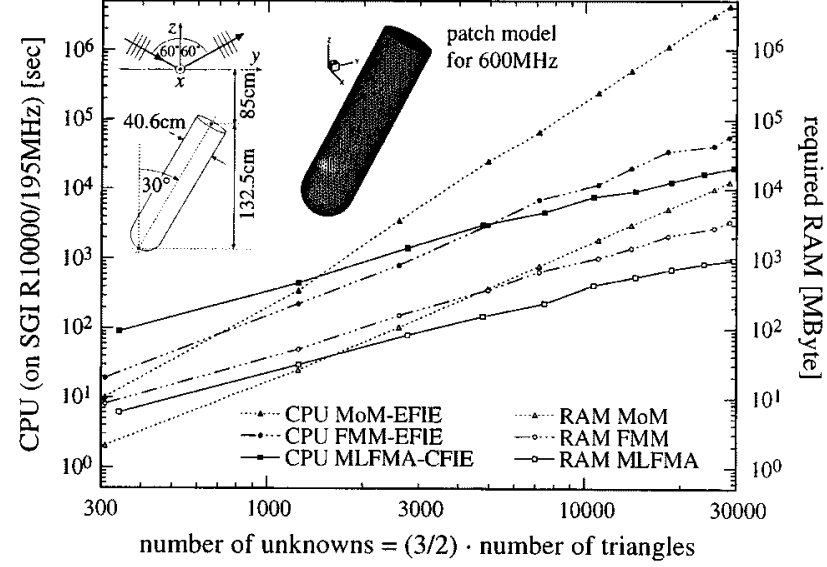

Fig. 6. Required RAM and overall CPU time (on SGI R10000/195 MHz) for bistatic RCS calculations as in Fig. 5 (polarimetric RCS matrix for 1800 scattering angles), for the frequency range $100-1000 \mathrm{MHz}$ (corresponding to $N=309-28539$ unknowns) using MoM (with LUD), FMM, and MLFMA. The CPU time for the MoM solution for $f>600 \mathrm{MHz}(N>10653)$ has been extrapolated.

the latter two calculated using an EFIE. Note that, although the UXO target is rotationally symmetric, the target-(half space) composite in Fig. 5 is not, and therefore cannot be modeled with the BOR-MoM software. Consequently, both the MoM and FMM computations employ the same triangular-patch basis functions as the MLFMA model. Using the coordinate system shown inset in Fig. 5, the plane wave is incident at $\phi_{\text {inc }}=-90^{\circ}$ and $\theta_{\text {inc }}=60^{\circ}$, and the bistatic fields are observed at $\theta_{\text {scat }}=$ $60^{\circ}$ and all $\phi_{\text {scat }}$. Due to bisectional symmetry, the cross-polarized fields should vanish at $\phi_{\text {scat }}= \pm 90^{\circ}$. From Fig. 5, we see that the three solutions are in good agreement, for all bistatic angles considered. We also note that, with Figs. 3 and 4 for comparison, the scattering of such a buried ordnance is quite small at the frequency $(600 \mathrm{MHz})$ considered, underscoring the difficulty of radar-based sensing of such targets. However, in a synthetic aperture radar (SAR) mode [37], the addition of signatures from multiple positions along the SAR aperture may provide a sufficient signature in the image for target detection. The MLFMA will be used in the future to perform such phenomenological studies as a function of target type, soil type, system bandwidth, aperture length, target orientation, and depression angle. Such a comprehensive set of data is difficult to acquire experimentally. The computations in Fig. 5 are characterized by 10653 unknowns (see Fig. 6 for RAM and CPU requirements).

Such phenomenological studies have motivated development of the MLFMA model, and it is therefore of interest to assess the CPU and RAM requirements of this technique versus the other algorithms presented in Fig. 5, namely, the MoM and FMM. In Fig. 6, we present the CPU and RAM requirements for bistatic results as in Fig. 5, covering the frequency range $100-1000 \mathrm{MHz}$ (corresponding to $N=309-28539$ unknowns). As expected [17]-[20], the MLFMA requires order $\mathrm{O}(N \lg N)$ RAM and CPU, while for the FMM these parameters are approximately order $\mathrm{O}\left(N^{3 / 2}\right)$. The slight undulations in the RAM/CPU complexity of the single-stage FMM are caused by a suboptimal clustering employed in our implementation of the FMM [28], [29]. We have found in general that the half-space 


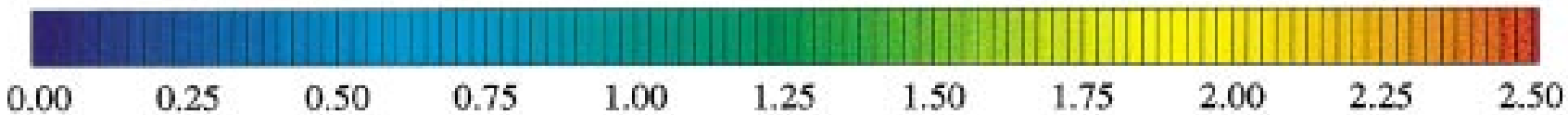

\section{magnitude of electric surface current relative to incident magnetic field}

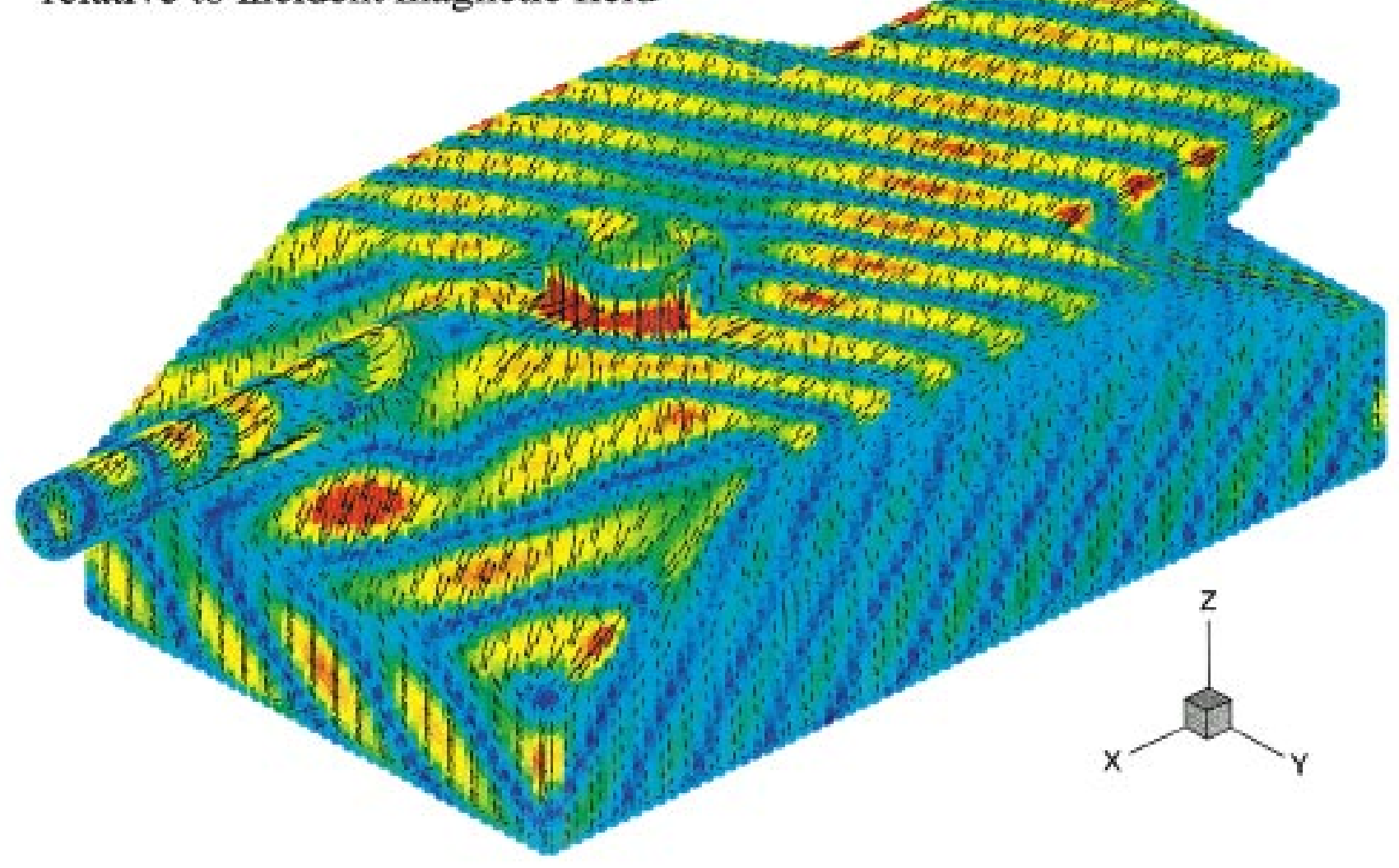

(a)

Fig. 7. Snapshot of the induced electric surface current on a model tank at a frequency of $700 \mathrm{MHz}$, for a plane wave incident from $\theta_{i n c}=60^{\circ}$ and $\phi_{i n c}=$ $30^{\circ}$. The magnitude of the surface current is coded in color, the arrows indicate the direction of the current flow. The five-level MLFMA solved a CFIE with $N=$ 59253 unknowns in about $7 \mathrm{~h}$ (both polarizations together) on a single SGI R10000/195 MHz processor using 2.68 GB (compared to 56.2 GB for a conventional MoM). (a) $V$-polarization incident.

MLFMA, as formulated here, requires typically 20-60\% more RAM than its free-space counterpart and approximately twice the CPU time (see also discussion Section III-C). Fig. 6 clearly demonstrates the efficacy of the MLFMA, vis-à-vis the MoM and FMM, this CPU and RAM enhancement being particularly important when numerous phenomenological studies are required of electrically large problems.

\section{Tank-Like Target}

In our final example, we present results of interest to radarbased sensing of vehicles over soil, by considering the tank-like target in Fig. 7. The target mesh is shown inset in Fig. 8 (for a frequency of $300 \mathrm{MHz}$ ), along with its associated dimensions. The target is $20 \mathrm{~cm}$ above the half space, here characterized as Yuma soil with $10 \%$ water content (see Fig. 2). The currents induced on the target are shown in Fig. 7, for $V$-polarization (a) and $H$-polarization (b) plane-wave excitation at $\theta_{\text {inc }}=60^{\circ}$ and $\phi_{\text {inc }}=30^{\circ}$ (see coordinate system inset in the figures). The arrows indicate the direction of the current flow, and the colors represent the normalized current magnitude. These results were computed via a five-level CFIE MLFMA with 59253 unknowns, requiring $7 \mathrm{~h}$ of total CPU run time (for both polarizations) on a SGI R10000/195 MHz processor, and 2.68 GB of RAM (a MoM solution requires 56.2 GB). The results presented here are for $700 \mathrm{MHz}$, corresponding to more than nine wavelengths along the length of the target.

The most striking aspect of the currents in Fig. 7 is the large electrical size of this target, reflected in the many undulations in current amplitude. An important distinction between the $V$-polarization and $H$-polarization results is reflected in the character of the currents induced on the flat portions of the target, which constitute one half of a dihedral reflector (the soil interface constituting the other half of the dihedral). Considering Fig. 7(b), we see the front and side faces of the target are characterized by a complicated interference pattern. This pattern reflects the superposition of currents induced directly by the incident plane wave (as if the target were in free space) plus the currents induced by the ground bounce of the incident fields. A similar pattern is manifested on the "cannon" portion of the target. By 


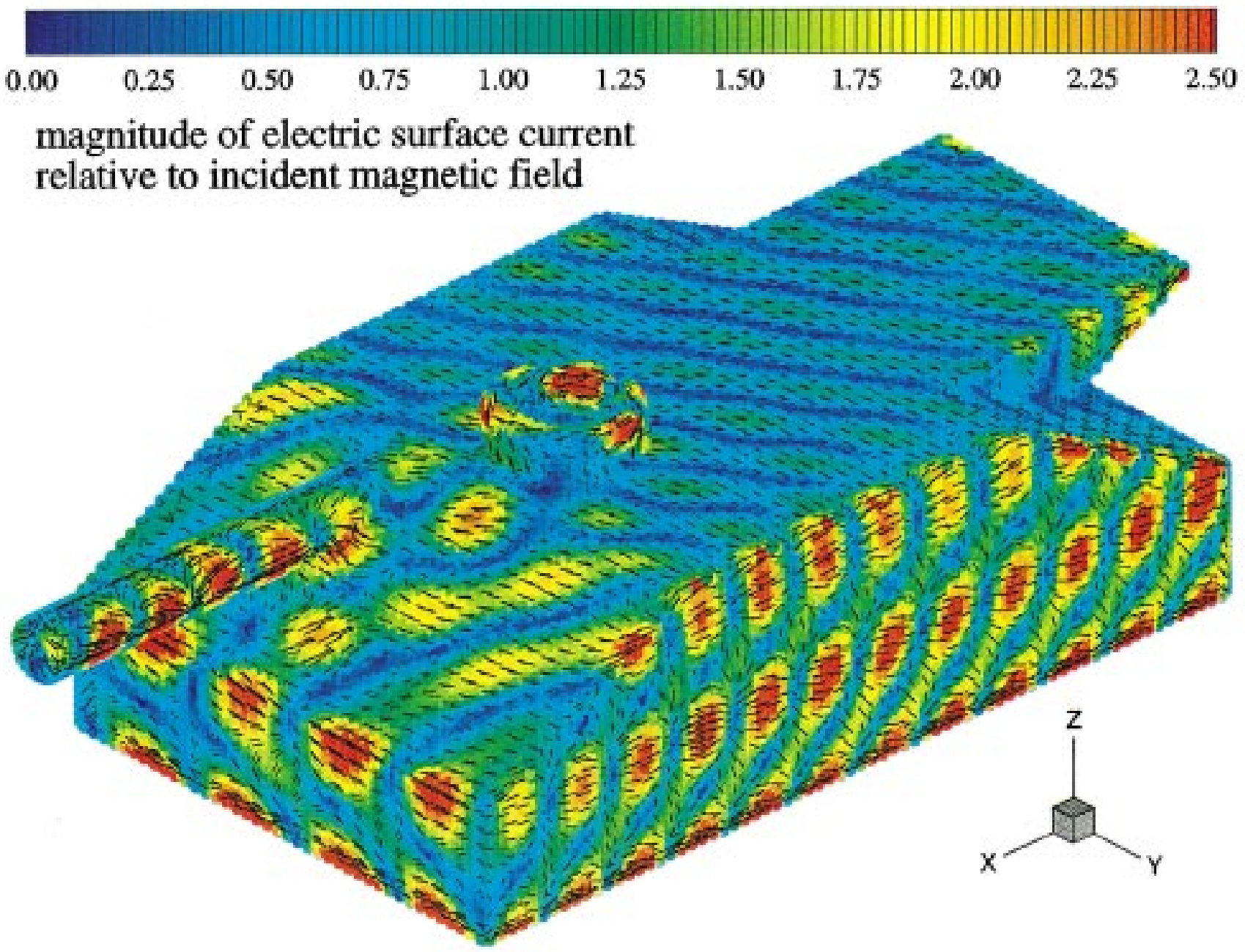

(b)

Fig. 7. (Continued.) Snapshot of the induced electric surface current on a model tank at a frequency of $700 \mathrm{MHz}$, for a plane wave incident from $\theta_{\text {inc }}=60^{\circ}$ and $\phi_{i n c}=30^{\circ}$. The magnitude of the surface current is coded in color, the arrows indicate the direction of the current flow. The five-level MLFMA solved a CFIE with $N=59253$ unknowns in about $7 \mathrm{~h}$ (both polarizations together) on a single SGI R10000/195 MHz processor using $2.68 \mathrm{~GB}$ (compared to $56.2 \mathrm{~GB}$ for a conventional MoM). (b) $H$-polarization incident.

contrast, this pattern is substantially reduced for the $V$-polarization in Fig. 7(a). As discussed when presenting the results in Fig. 3, the near-Brewster excitation results in significant penetration of the incident wave into the soil, markedly reducing the strength of the ground bounce.

One of the fundamental challenges of detecting large man-made targets similar to that considered in Fig. 7 is the strong signature variation as a function of target-sensor orientation. Such targets are generally concealed, and therefore, the target aspect is generally unknown a priori, significantly complicating radar-based target detection and identification. The modeling of such phenomena is particularly important to the development of signal processing algorithms, since a comprehensive set of measurements, for all target-sensor orientations, frequencies, incident angles, polarizations, and soil types are generally difficult to attain.

In Fig. 8, we plot the monostatic RCS of the tank-like target, for the copolarized fields, at a frequency of $300 \mathrm{MHz}$ and an angle $\theta=60^{\circ}$. These results involved $N=13734$ unknowns, and the four-level MLFMA required $646 \mathrm{MB}$ of RAM (an
MoM solution requires $3.1 \mathrm{~GB}$ ) and $129 \mathrm{~h}$ of CPU on one SGI $\mathrm{R} 10000 / 195 \mathrm{MHz}$ processor (for all 360 monostatic angles). The results were performed consecutively in azimuth, sampling $\phi$ at an increment of $\Delta \phi=1^{\circ}$. To accelerate convergence of the conjugate-gradient solver, the current solution from the previous azimuthal angle was used as the initial guess for the subsequent solution, reducing the number of iterations per angle and polarization from approximately 90 (for zero initial guess) to an average of approximately 35 . As indicated when discussing Fig. 3, the model's ability to satisfy reciprocity is one indicator of the dynamic range over which the results can be trusted. The cross-polarized fields for this target (not shown here) indicate that the results are highly accurate down to approximately $-10 \mathrm{dBsm}$. Further accuracy can be achieved, if desired, by evaluating the Green's function more accurately for the near MLFMA terms (see the discussion associated with Fig. 3), by reducing the relative residual error at which the iterative solver is deemed to converge (for Figs. 8 and 9 we used $1 \%$ ) and by using more terms in the various plane wave representations in the MLFMA (i.e., increasing the number of 


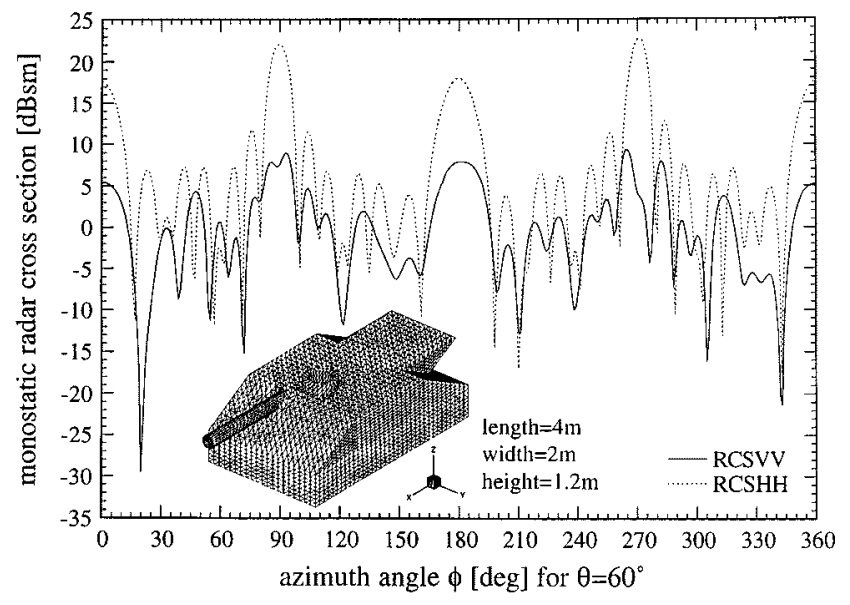

Fig. 8. Radar cross section (here we only plot VV and $\mathrm{HH}$ ) of a model tank with $N=13734$ unknowns (see inset) situated above Yuma soil of $10 \%$ water content (see Fig. 2) at a frequency of $300 \mathrm{MHz}$. Monostatic RCS is plotted for $\theta=60^{\circ}$ ( $30^{\circ}$ from grazing) and varying azimuth angles $\phi$. The four-level MLFMA required a total of $646 \mathrm{MB}$ of RAM (compared to $3.1 \mathrm{~GB}$ for a conventional MoM) and approximately $129 \mathrm{~h}$ of CPU time (on a single SGI $\mathrm{R} 10000 / 195 \mathrm{MHz}$ processor) for 360 monostatic angles.

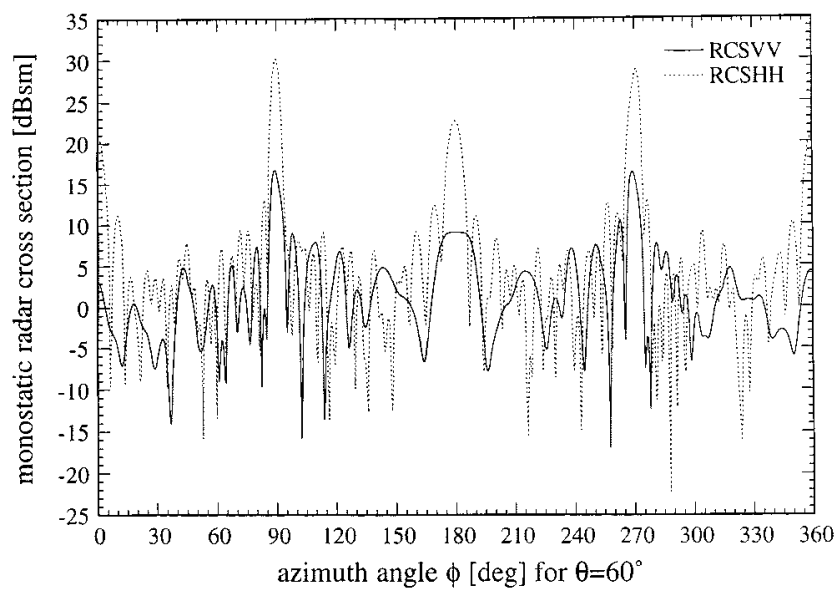

Fig. 9. As in Fig. 8, but for a frequency of $700 \mathrm{MHz}$ and $N=59253$ unknowns (the mesh in Fig. 8 was refined). The five-level MLFMA required $2.68 \mathrm{~GB}$ of RAM (compared to 56.2 GB for a conventional MoM solution) and $1022 \mathrm{~h}$ of total CPU time (on a single SGI R10000/195 MHz processor) for 720 monostatic angles.

terms $L$ in the addition theorem expansion beyond the required $L$ for an error less than $1 \%$, which has been applied for all examples presented here) [17]-[20].

In Fig. 9, we again consider the monostatic RCS of the tank-like target, now computed at a frequency of $700 \mathrm{MHz}$. For these results, the azimuthal angle was sampled at a rate of $\Delta \phi=0.5^{\circ}$, to accurately capture the faster variation of the RCS at higher frequencies. For these computations the target was characterized by $N=59253$ unknowns, and the five-level MLFMA required $2.68 \mathrm{~GB}$ of RAM. As in Fig. 8, we consider $\theta=60^{\circ}$. The computation of the full-polarimetric RCS (i.e., $\mathrm{VV}, \mathrm{VH}, \mathrm{HV}$, and $\mathrm{HH}$ ), at 720 different angles, required 1022 hours of total CPU time on a single SGI R10000/195 MHz processor. The RAM and $\mathrm{CPU}$ requirements (compared to the $300 \mathrm{MHz}$ case) are consistent with the theoretical complexity of $\mathrm{O}(N \lg N)$, when noting that the number of angles in the $700 \mathrm{MHz}$ calculation was doubled. As expected, relative to the $300 \mathrm{MHz}$ example of Fig. 8, the RCS results are characterized by substantially faster variation with azimuthal angle.

\section{CONCLUSIONS}

An extension of the MLFMA has been presented for the analysis of electromagnetic scattering from electrically large perfect conductors situated in the presence of a half space. The structure of the half-space MLFMA is similar in many ways to its freespace counterpart [17]-[20]. In particular, we exploit properties of the free space Green's function to compute far (expansion function)-(testing function) interactions cumulatively through use of a multi-level clustering procedure [17]-[20]. Within the context of nonnearby cluster computations, interaction with the half space is accounted for approximately via polarization-dependent images located at real spatial locations. Hence, the first escalation in complexity of the half-space MLFMA relative to the free-space version is the introduction of image clusters, located in real space. In addition, the near interactions in the halfspace MLFMA are treated via a rigorous analysis of the dyadic Green's function, here computed via the method of complex images [25]-[27]. We have found such a rigorous analysis of the near interactions essential for generating accurate results.

The MLFMA was calibrated by considering a special problem applicable to a MoM solution tailored to a BOR. This provides a good test, because the BOR-MoM solution is numerically rigorous, and because the BOR-MoM and MLFMA solutions employ distinct basis functions. The agreement between the MLFMA and BOR-MoM results was excellent, for all examples considered. Results were also presented for a UXO embedded in a lossy half space. For that problem the accuracy of the MLFMA was favorably compared with MoM and FMM solutions. Moreover, CPU and RAM comparisons clearly demonstrated the efficacy of the MLFMA technique, vis-à-vis the MoM and FMM. The final example considered a tank-like target, for which several results were presented.

The results for scattering from the tank-like target were insightful, while also demonstrating that, for such a large target, the memory requirements of the MLFMA are substantially smaller than those of the MoM. However, these results also clearly demonstrate that significant work is still required on the development of modeling algorithms for wideband, multi-aspect scattering from such targets. In particular, we noted that the multi-aspect computations in Figs. 8 and 9 required substantial CPU (although much less than would be required using a MoM model), and in practice, we would likely also require data over a wide frequency range. These observations point to directions for future research. The half-space MLFMA represents a numerically rigorous analysis of Maxwell's equations, within the context of the Green's-function approximation (for far interactions) discussed previously. Consequently, we can use the MLFMA results at high frequencies (such as in Fig. 9) to examine the accuracy of approximate, asymptotic solutions. The MLFMA can then be used to fill the frequency gap for which the MoM is intractable and for which asymptotic solutions are inappropriate. In addition, all results presented here were run on a single processor. It is of interest to develop a 
scaleable, parallel version of the half-space MLFMA. Finally, in all MLFMA results, we have employed the RWG triangular-patch basis functions [7]. These basis functions are quite general, but for electrically large problems, they result in a very significant number of unknowns. Researchers have, within the context of MoM, developed hybrid techniques that combine subsectional basis functions with asymptotic traveling- wave and edge-diffraction basis functions [40]. The use of such basis functions substantially reduces the number of unknowns, reducing the problem size. It is of interest to incorporate such techniques into the MLFMA framework.

\section{REFERENCES}

[1] S. Vitebskiy, K. Sturgess, and L. Carin, "Short-pulse plane-wave scattering from buried perfectly conducting bodies of revolution," IEEE Trans. Antennas Propagat., vol. 44, pp. 143-151, Feb. 1996.

[2] N. Geng and L. Carin, "Wideband electromagnetic scattering from a dielectric BOR buried in a layered lossy, dispersive medium," IEEE Trans. Antennas Propagat., vol. 47, pp. 610-619, Apr. 1999.

[3] N. Geng, M. Ressler, and L. Carin, "Wideband VHF scattering from a trihedral reflector situated above a lossy dispersive half space,", to be published.

[4] J. M. Bourgeois and G. S. Smith, "A fully three-dimensional simulation of a ground-penetrating radar: FDTD theory compared with experiment," IEEE Trans. Geosci. Remote Sensing, vol. 34, pp. 36-44, Jan. 1996.

[5] - "A complete electromagnetic simulation of the separated-aperture sensor for detecting buried land mines," IEEE Trans. Antennas Propagat., vol. 46, pp. 1419-1426, Oct. 1998.

[6] H. S. Chang and K. K. Mei, "Scattering of electromagnetic waves by buried and partly buried bodies of revolution," IEEE Trans. Geosci. Remote Sensing, vol. GE-23, pp. 596-605, 1985.

[7] S. M. Rao, D. R. Wilton, and A. W. Glisson, "Electromagnetic scattering from surfaces of arbitrary shape," IEEE Trans. Antennas Propagat., vol. 30, pp. 409-418, May 1982.

[8] K. A. Michalski and D. Zheng, "Electromagnetic scattering and radiation by surfaces of arbitrary shape in layered media, Parts I and II," IEEE Trans. Antennas Propagat., vol. 38, pp. 335-352, Mar. 1990.

[9] A. F. Peterson, S. L. Ray, and R. Mittra, Computational Methods for Electromagnetics. Piscataway, NJ: IEEE Press, 1998.

[10] J. M. Jin, The Finite Element Method in Electromagnetics. New York, NY: Wiley, 1993

[11] A. Taflove, Computational Electromagnetics-The Finite-Difference Time-Domain Method. Boston, MA: Artech House, 1995.

[12] T. Dogaru and L. Carin, "Application of multi-resolution time-domain to two-dimensional electromagnetic scattering problems,", submitted for publication.

[13] A. Taflove, Ed., Advances in Computational Electromagnetics-The Finite-Difference Time-Domain Method. Norwood, MA: Artech House, 1998.

[14] R. Coifman, V. Rokhlin, and S. Wandzura, "The fast multipole method for the wave equation: A pedestrian prescription," IEEE Antennas Propagat. Mag., vol. 35, pp. 7-12, June 1993

[15] J. M. Song and W. C. Chew, "Fast multipole method solution using parametric geometry," Microw. Opt. Technol. Lett., vol. 7, pp. 760-765, Nov. 1994.

[16] A. Brandt, "Multilevel computations of integral transforms and particle interactions with oscillatory kernels," Comput. Phys. Commun., vol. 65, pp. 24-38, 1991.

[17] J. M. Song and W. C. Chew, "Multilevel fast multipole algorithm for solving combined field integral equations of electromagnetic scattering," Microw. Opt. Technol. Lett., vol. 10, pp. 14-19, Sept. 1995.

[18] J. M. Song, C. C. Lu, W. C. Chew, and S. W. Lee, "Fast Illinois solver code (FISC)," IEEE Trans. Antennas Propagat. Mag., vol. 40, pp. 27-33, June 1998.

[19] J. M. Song, C. C. Lu, and W. C. Chew, "Multilevel fast multipole algorithm for electromagnetic scattering by large complex objects," IEEE Trans. Antennas Propagat., vol. 45, pp. 1488-1493, Oct. 1997.

[20] X. Q. Sheng, J. M. Jin, J. Song, W. C. Chew, and C. C. Lu, "Solution of combined-field integral equation using multilevel fast multipole algorithm for scattering by homogeneous bodies," IEEE Trans. Antennas Propagat., vol. 46, pp. 1718-1726, Nov. 1998.
[21] M. Abramowitz and I. A. Stegun, Handbook of Mathematical Functions. New York: Dover, 1970

[22] L. Gürel and M. I. Aksun, "Electromagnetic scattering solution of conducting strips in layered media using the fast multipole method," IEEE Microwave Guided Wave Lett., vol. 6, pp. 277-279, Aug. 1996.

[23] J. S. Zhao, W. C. Chew, C. C. Lu, E. Michielssen, and J. Song, "Thinstratified medium fast-multipole algorithm for solving microstrip structures," IEEE Trans. Microw. Theory Tech., vol. 46, pp. 395-403, Apr. 1998.

[24] Y. Rahmat-Samii, R. Mittra, and P. Parhami, "Evaluation of Sommerfeld integralsor lossy half-space problems," Electromagnetics, vol. 1, no. 1, pp. $1-28,1981$.

[25] J. J. Yang, Y. L. Chow, and D. G. Fang, "Discrete complex images of a three-dimensional dipole above and within a lossy ground," Proc. Inst. Elect. Eng. H, vol. 138, pp. 319-326, Aug. 1991.

[26] R. M. Shubair and Y. L. Chow, "A simple and accurate complex image interpretation of vertical antennas present in contiguous dielectric halfspaces," IEEE Trans. Antennas Propagat., vol. 41, pp. 806-812, June 1993.

[27] M. I. Aksun, "A robust approach for the derivation of closed-form Green's functions," Trans. Microw. Theory Tech., vol. 44, pp. 651-658, May 1996.

[28] N. Geng, A. Sullivan, and L. Carin, "Fast multipole method for scattering from 3D PEC targets situated in a half-space environment," Microw. Opt. Technol. Lett., vol. 21, pp. 399-405, June 1999.

[29] —_, "Fast multipole method for scattering from an arbitrary PEC target above or buried in a lossy half space,", to be published.

[30] I. V. Lindell, Methods for Electromagnetic Field Analysis. Piscataway, NJ: IEEE Press, 1995.

[31] D. R. Wilton, S. M. Rao, A. W. Glisson, D. H. Schaubert, O. M. Al-Bundak, and C. M. Butler, "Potential integrals for uniform and linear source distributions on polygonal and polyhedral domains," IEEE Trans. Antennas Propagat., vol. 32, pp. 276-281, Mar. 1984.

[32] R. E. Hodges and Y. Rahmat-Samii, "The evaluation of MFIE integrals with the use of vector triangle basis functions," Microw. Opt. Technol. Lett., vol. 14, pp. 9-14, Jan. 1997.

[33] A. D. McLaren, "Optimal numerical integration on a sphere," Math. Comput., vol. 17, pp. 361-383, 1963.

[34] T. K. Sarkar and E. Arvas, "On a class of finite step iterative methods (conjugate directions) for the solution of an operator equation arising in electromagnetics," IEEE Trans. Antennas Propagat., vol. GE-33, pp. 1058-1066, Oct. 1985.

[35] Y. Saad, Iterative Methods for Sparse Linear Systems. Boston, MA: PWS, 1996.

[36] N. M. Nachtigal, S. C. Reddy, and L. N. Trefethen, "How fast are nonsymmetric matrix iterations?," SIAM J. Matrix Anal. Appl., vol. 13, pp. 778-795, July 1992.

[37] S. Vitebskiy, L. Carin, M. A. Ressler, and F. H. Le, "Ultra-wideband, short-pulse ground-penetrating radar: Simulation and measurement," IEEE Trans. Geosci. Remote Sensing, vol. 35, pp. 762-772, May 1997.

[38] L. Carin, R. Kapoor, and C. E. Baum, "Polarimetric SAR imaging of buried landmines,", to be published.

[39] M. L. Van Blaricum and R. Mittra, "A technique for extracting the poles and residues of a system directly from its transient response," IEEE Trans. Antennas Propagat., vol. 23, pp. 777-781, Nov. 1975.

[40] A. Sullivan and L. Carin, "A hybrid technique combining the moment method with physical optics and uniform asymptotics for scattering from 2D cylinders," Microw. Opt. Technol. Lett., vol. 21, pp. 117-121, Apr. 1999.

Norbert Geng (S'91-M'96) was on born May 14, 1965 in Lauchringen, Germany. He received the Dipl.-Ing. and Dr.-Ing. degrees in electrical engineering from the University of Karlsruhe, Germany, in 1991 and 1996, respectively.

From 1991 to 1996, he was with the Institute for Microwaves and Electronics, University of Karlsruhe, working on full-wave propagation modeling for radio communications systems. From 1997 to 1998, he was with the Department of Electrical and Computer Engineering, Duke University, Durham, NC, as a Visiting Postdoctoral Researcher. From 1998 to 1999, he was with the University of Karlsruhe. His research at both of these universities focused on numerical techniques in computational electromagnetics for planar-stratified media. Since January 2000, he has been with SIEMENS Corporate Technology, Munich, Germany, as a Research Scientist on the design of broadband wireless communications systems.

Dr. Geng received the Mannesmann Innovation Award in 1997 for his Ph.D. dissertation on this subject. 
Anders Sullivan (M'93) was born on August 9, 1963, in Staten Island, NY. He received the B.S. and M.S. degrees in aerospace engineering from the Georgia Institute of Technology, Atlanta, GA, in 1985 and 1987, respectively, and the Ph.D. degree in electromagnetics from Polytechnic University, Brooklyn, NY, in 1997.

From 1988 through May 1998, he was with the Air Force Research Lab, Eglin Air Force Base, FL. From June 1998 to September 1999, he was with the Electrical Engineering Department, Duke University, Durham, NC, as a Research Associate. Since September 1999, he has been with the Army Research Lab, Adelphi, MD. His current research interests include modeling complex targets and short-pulse scattering.

Dr. Sullivan is a member of the Tau Beta Pi and Sigma Gamma Tau honor societies.
Lawrence Carin (SM'96) was born on March 25, 1963 in Washington, DC. He received the B.S., M.S., and Ph.D. degrees in electrical engineering from the University of Maryland, College Park, in 1985, 1986, and 1989, respectively.

In 1989, he joined the Electrical Engineering Department, Polytechnic University, Brooklyn, NY, as an Assistant Professor, and became an Associate Professor in 1994. Since September 1995, he has been with the Electrical Engineering Department, Duke University, Durham, NC, where he is an Associate Professor. He is the Principal Investigator on a Multidisciplinary University Research Initiative (MURI) on demining. His current research interests include short-pulse scattering, subsurface sensing, and wave-based signal processing.

Dr. Carin is currently an Associate Editor of the IEEE TRANSACTIONS ON Antennas and Propagation. He is a member of Tau Beta Pi and Eta Kappa $\mathrm{Nu}$ 